\title{
Kappa-Symmetry of Green-Schwarz Actions in Coset Superspaces
}

\author{
I. N. McArthurf \\ Department of Physics, The University of Western Australia \\ Nedlands, W.A. 690\%. Australia
}

\begin{abstract}
The nature of $\kappa$-symmmetry transformations is examined for $p$-branes embedded in a class of coset superspaces $G / H$, where $G$ is an appropriate supergroup and $H$ is the Lorentz subgroup. It is shown that one of the conditions $\delta Z^{M} E_{M}{ }^{a}=0$ which characterizes $\kappa$-symmetry transformations arises very naturally if they are implemented in terms of a right action of a subgroup of the supergroup $G$ on the supergroup elements which represent the coset. Unlike the global left action of $G$ on $G / H$ (which gives rise to supersymmetry on the coset superspace), there is no canonically defined right action of $G$ on $G / H$. However, an interpretation of this right action involving an enlargement of the isotropy group from the Lorentz subgroup to a subgroup of $G$ with generators which include some of the fermionic generators of $G$ is suggested. Closure of the generators of this larger subgroup under commutation leads to the usual "brane scan" for $p$-branes which have bosonic degrees of freedom which are worldvolume scalars.
\end{abstract}

\footnotetext{
${ }^{1}$ E-mail: mcarthur@physics.uwa.edu.au
} 


\section{Introduction}

Kappa symmetry is a local fermionic symmetry of covariant Green-Schwarz actions [1] for $p$-branes. It is a generalization of local fermionic symmetries discovered for massive [2] and massless [3] superparticle actions. The presence of the $\kappa$-symmetry in the Green-Schwarz action for a $p$-brane ensures that only half of the supersymmetry generators are realized nonlinearly, as required for low energy effective actions describing the long wavelength excitations of BPS solitons [4, 5]. The standard procedure for forming $\kappa$ symmetric Green-Schwarz actions in flat superspace is the "nonlinear sigma model approach" [6, []. The kinetic term is a natural generalization of the Nambu action, to which is added a Wess-Zumino term formed by integrating a closed form in superspace over an embedded manifold whose boundary is the worldvolume of the $p$-branef . Global supersymmetry of the action is ensured by constructing both pieces of the action in terms of the vielbein on flat superspace. The $\kappa$-symmetry is then a field dependent local fermionic transformation for which the variations of the the kinetic term and the WessZumino term can be made to cancel by appropriate choice of the normalization of the Wess-Zumino term. This procedure has recently been extended to the case of superspace backgrounds which are more general cosets than flat superspace [8].

Progress has been made in achieving a geometric understanding of kappa symmetry using the formalism of super-embeddings [9], based on doubly supersymmetric approaches to superstrings which incorporate both manifest worldvolume and spacetime supersymmetries [10] (for a recent review of superembeddings including a comprehensive list of references, see [11]). The worldvolume of the $p$-brane is extended to a supermanifold which is embedded into the target superspace in such a way that the odd subspace of the worldvolume tangent space is a subset of the odd subspace of tangent space to the target superspace. In such a formulation, the fermionic kappa symmetry can be related to the worldvolume supersymmetry.

It has been noted in the past that in flat superspace, the $\kappa$-symmetry transformation of the bosonic superspace coordinate is like a "wrong-signed" supersymmetry transformation [12]. In terms of the construction of flat superspace as the left coset $G / H$ of the super-Poincaré group $G$ with the

\footnotetext{
${ }^{2}$ Only $p$-branes which have bosonic degrees of freedom which are worldvolume scalars will be considered in this paper.
} 
Lorentz subgroup as the isotropy group $H$ [13], and in which supersymmetry transformations of the superspace coordinates are achieved by the canonically defined left action of the superalgebra on the coset superspace, this is an indication that $\kappa$-symmetry transformations should be related to the right action of the superalgebra on the same coset. In the Hamiltonian formulation for $p$-branes embedded in flat superspace, de Azcárraga, Izquierdo and Townsend [14] showed that the fermionic constraints (some of which are first class, corresponding to the existence of local $\kappa$-symmetry) are analogous to supercovariant derivatives in superspace, which generate right supertranslations. Since left and right supertranslations commute, this provides a means to implement fermionic constraints which are consistent with the global supersymmetry generated by left supertranslations. More recently Bars, Deliduman and Minic [15] have implemented $\kappa$-symmetry transformations for a number of non-flat coset superspaces in terms of a local right action.

The aim of this paper is to point out that for a general class of coset superspaces, $\kappa$-symmetry transformations do indeed have a natural interpretation in terms of a local right action, as opposed to the global left action which gives rise to the supersymmetry. In particular, after a review of the relevant properties of coset spaces in $\S 2$ and a discussion of relevant coset superspaces in the first part of $\S 3$, it will be shown that the criterion

$$
\delta Z^{M} E_{M}^{a}=0
$$

which characterizes $\kappa$-symmetry transformations in curved superspace 16, 17] is very naturally implemented for coset superspaces $G / H$ in terms of a local right action of a part of the supergroup $G$. Although only the left action of the supergroup $G$ on the coset superspace $G / H$ is canonically defined (this is the usual nonlinear realization of $G$ on $G / H$, which is independent of a particular parameterization of the coset), it is argued that the local right action associated with the $\kappa$-symmetry transformations is also well-defined, and should be interpreted as giving rise to an enlargement of the isotropy group $H$ from the Lorentz subgroup to a larger subgroup $\tilde{H}$ which includes fermionic generators. This is consistent with the role of $\kappa$-symmetry in covariant Green-Schwarz actions, which is to ensure that although there appear to be Goldstone fermions associated with all the supersymmetry generators, in fact only half of them are physical, meaning that only half of the supersymmetry is realized nonlinearly, with the rest of the supersymmetry being 
realized linearly on the physical degrees of freedom. In $\S 4$, this interpretation is expanded to include the worldvolume diffeomorphisms, which also eliminate some of the apparent Goldstone degrees of freedom in covariant Green-Schwarz actions. It is argued in $\S 5$ that consistency of this picture requires that the generators of the right actions associated with the $\kappa$-symmetry transformations and the worldvolume diffeomorphisms must form a closed superalgebra, from which emerge the usual restrictions on relationship between the dimension of the worldvolume of a $p$-brane and the dimension of the spacetime into which it is embedded (the "brane-scan" [18).

\section{Some Preliminaries}

We begin by reviewing relevant details of coset spaces[ ${ }^{[3}$ and nonlinear realizations. Consider a Lie algebra $G$ with generators $T_{\mathcal{A}}$ satisfying the (possibly graded) commutation relations

$$
\left[T_{\mathcal{A}}, T_{\mathcal{B}}\right]=i f_{\mathcal{A B}}{ }^{\mathcal{C}} T_{\mathcal{C}}
$$

Let $T_{\bar{I}}$ denote the generators of a subalgebra $H$ of $G$ (the "unbroken" generators), and $T_{A}$ denote the remaining generators of $G$ (the "broken" generators), and assume that the $T_{A}$ provide a representation of $H$ under commutationf. A "slice" through $G$ locally isomorphic to $G / H$ can be parameterized in the form

$$
g(Z)=\exp \left(i Z^{M} \delta_{M}^{A} T_{A}\right),
$$

with the $Z^{M}$ furnishing local coordinates on $G / H$. There is a canonical global left action of $G$ on $G / H$ under which the point with coordinates $Z$ is mapped by fixed $g \in G$ into the point with coordinates $Z^{\prime}$ determined by

$$
g \cdot g(Z)=g\left(Z^{\prime}\right) \cdot h(Z, g)
$$

Here, $h(Z, g)$ is the compensating $H$-transformation required to bring $g . g(Z)$ back onto the slice (11) isomorphic to $G / H$. The realization of the group $G$ on the coordinates $Z$ is nonlinear.

\footnotetext{
${ }^{3}$ The cosets considered in this paper are always left cosets.

${ }^{4}$ The index conventions to be used are as follows: indices $\bar{I}, \bar{J}, \bar{K}, \ldots$ are used for the generators of $H$; indices $A, B, C, \ldots$ are used for generators of $G$ not in $H$; and $M, N, \ldots$ are used for coordinates on $G / H$.
} 
The Cartan one-form $g(Z)^{-1} d g(Z)$ defined on $G / H$ takes values in the complexification of the Lie algebra of $G$ and can be decomposed as

$$
g(Z)^{-1} d g(Z)=i d Z^{M} E_{M}^{A}(Z) T_{A}+i d Z^{M} \Omega_{M}^{\bar{I}}(Z) T_{\bar{I}}
$$

These forms are invariant the global left action of $G$ on $G / H$ since $d(g . g(Z))=$ $g . d g(Z)$ if $g$ is constant. Under the local right action $g(Z) \rightarrow g(Z) . h(Z)$ with $h(Z)=e^{i \epsilon^{\bar{I}}(Z) T_{\bar{I}}} \in H$, the forms $E^{A}(Z)=d Z^{M} E_{M} A(Z)$ transform as a vielbein on the tangent bundle to $G / H$, while $\Omega^{\bar{I}}(Z)=d Z^{M} \Omega_{M}{ }^{\bar{I}}(Z)$ transforms as a connection on this bundle (see, for example, [19]). The Maurer Cartan equations $0=d\left(g(Z)^{-1} d g(Z)\right)+g(Z)^{-1} d g(Z) \wedge g(Z)^{-1} d g(Z)$ can be written in the form

$$
\begin{aligned}
-\frac{1}{2} E^{C}(Z) \wedge E^{B}(Z) f_{B C}{ }^{A} & =d E^{A}(Z)+\Omega^{\bar{I}}(Z) \wedge E^{B}(Z) f_{B \bar{I}}{ }^{A} \\
-\frac{1}{2} E^{C}(Z) \wedge E^{B}(Z) f_{B C}{ }^{\bar{I}} & =d \Omega^{\bar{I}}(Z)+\frac{1}{2} \Omega^{\bar{K}}(Z) \wedge \Omega^{\bar{J}}(Z) f_{\bar{J} \bar{K}}{ }^{\bar{I}},
\end{aligned}
$$

so that the left-hand sides of these expressions are respectively the torsion and curvature of the coset space.

Replacing $d$ by $\delta$ in (3), an infinitesimal variation $\delta Z^{M}$ of the coordinate $Z^{M}$ on $G / H$ will induce a variation $\delta g(Z)$ of the representative group element $g(Z)$ which satisfies

$$
g(Z)^{-1} \delta g(Z)=i \delta Z^{M} E_{M}^{A}(Z) T_{A}+i \delta Z^{M} \Omega_{M}^{\bar{I}}(Z) T_{\bar{I}}
$$

Consider now the right action of the group $G$ on the representatives $g(Z)$ of the points in the coset $G / H$. In particular, consider

$$
g(Z) \rightarrow g(Z) \cdot e^{i\left(v^{A}(Z) T_{A}+w^{\bar{I}}(Z) T_{\bar{I}}\right)} .
$$

Since the new group element will lie on the $H$-orbit of some point on the slice, one has

$$
g(Z) \cdot e^{i\left(v^{A}(Z) T_{A}+w^{\bar{I}}(Z) T_{\bar{I}}\right)}=g\left(Z^{\prime}\right) \cdot h(v, w, Z)
$$

for some $h(v, w, Z) \in H$. In infinitesimal form one obtains

$$
\left.g\left(Z^{\prime}\right)=g(Z) \cdot\left(1+i v^{A}(Z) T_{A}+i w^{\bar{I}}(Z) T_{\bar{I}}\right)\right) \cdot\left(1-i \phi^{\bar{I}}(v, w, Z) T_{\bar{I}}\right)
$$

\footnotetext{
${ }^{5}$ Note that there is no canonical right action of the group $G$ on the (left) coset space $G / H$, unlike the case of the left action of the group. The right action considered here is in general dependent on the choice of the slice $g(Z)$.
} 
where $\phi^{\bar{I}}(v, w, Z)$ is linear in $v^{A}(Z)$ and $w^{\bar{I}}(Z)$. This yields

$$
g(Z)^{-1} \delta g(Z)=i v^{A}(Z) T_{A}+i\left(w^{\bar{I}}(Z)-\phi^{\bar{I}}(v, w, Z)\right) T_{\bar{I}}
$$

Comparing (5) and (7),

$$
v^{A}(Z)=\delta Z^{M} E_{M}^{A}(Z)
$$

which allows $\delta Z^{M}$ to be computed given $v^{A}(Z)$. The remaining relation

$$
\delta Z^{M} \Omega_{M}^{\bar{I}}(Z)=w^{\bar{I}}(Z)-\phi^{\bar{I}}(v, w, Z)
$$

determines the compensating Lorentz transformation $\phi^{\bar{I}}$.

\section{Coset Superspaces}

The analysis of $\kappa$-symmetric Green-Schwarz actions for $p$-branes in an generic ten or eleven dimensional superspace backgrounds [17, 21] is complicated by the fact that there is no general prescription for the construction of the superfields which correspond to a given bosonic background [20]. However, the fact that flat superspaces can be considered as cosets of the super-Poincaré group with the Lorentz subgroup as the stability group [22] allows a geometric formulation of the Green-Schwarz action in terms of a nonlinear sigma model on the coset space [6, 5, 23]. Recently, this construction has been extended to the case of superspaces whose bosonic parts correspond to the near horizon geometries of various $p$-brane solitons, which are of the form $A d S_{p+2} \times S^{d-p-2}$. The corresponding superspaces can be realized as coset spaces $G / H$ for some choice of supergroup $G$ and bosonic stability group $H$, particular cases of interest being

$$
\begin{aligned}
& A d S_{5} \times S^{5} \cong S U(2,2 \mid 4) /(S O(1,4) \times S O(5)) \\
& A d S_{4} \times S^{7} \cong O S p(8 \mid 4) /(S O(1,3) \times S O(7)) \\
& A d S_{7} \times S^{4} \cong O S p(6,2 \mid 4) /(S O(1,6) \times S O(4)) .
\end{aligned}
$$

These correspond respectively to the $D 3$ brane in $d=10$ IIB supergravity [8], the $M 2$ brane in $d=11$ supergravity, and the $M 5$ brane in $d=11$ supergravity 24, 25].

The coset superspaces $G / H$ above are based on supergroups $G$ with generators which will be denoted $P_{a}, Q_{\alpha}$ and $J_{a b}$. The $J_{a b}$ are generators of a 
Lorentz subgroup $H$, under which the even generators $P_{a}$ transform as a vector and the odd generators $Q_{\alpha}$ transform as spinors:

$$
\begin{aligned}
{\left[J_{a b}, P_{c}\right] } & =\frac{i}{2}\left(\eta_{a c} \delta_{b}{ }^{d}-\eta_{b c} \delta_{a}{ }^{d}\right) P_{d} \\
{\left[J_{a b}, Q^{\alpha}\right] } & =\frac{i}{8}\left[\Gamma_{a}, \Gamma_{b}\right]_{\beta}^{\alpha} Q^{\beta} .
\end{aligned}
$$

The remaining (anti)commutation relations for the Lie algebra of the supergroup $G$ will not be specified, except to require that the algebra has a contraction to the usual (possibly extended) supersymmetry algebra, as a result of which the anticommutator of odd generators takes the form

$$
\left\{Q_{\alpha}, Q_{\beta}\right\}=-2\left(C \Gamma^{a}\right)_{\alpha \beta} P_{a}+i f_{\alpha \beta}^{a b} J_{a b}
$$

This structure encompasses the super-Poincaré group appropriate to the construction of flat superspaces, as well as the more general supergroups in the coset superspaces (10). The coset superspaces can be parameterized in the form

$$
g(Z)=e^{i\left(x^{m} \delta_{m}{ }^{a} P_{a}+\bar{\theta} Q\right)} \equiv e^{i Z^{M} \delta_{M}^{A} T_{A}},
$$

where $Z^{M}=\left(x^{m}, \theta^{\alpha}\right)$, and $T_{A}=\left(P_{a}, Q_{\alpha}\right)$ are the "broken" generators (and $T_{\bar{I}}=\left(J_{a b}\right)$ are the "unbroken" generators $)$.

Evidence is now provided for the identification of the $\kappa$-symmetries of the Green-Schwarz actions in these coset superspaces $G / H$ with a right action? of the the supergroup $G$. The canonically defined global action of an element $g \in G$ on the coset superspace is determined the left action $g \cdot g(Z)$, as in (2). A Green-Schwarz action is automatically invariant under this group action since it is constructed from the Cartan forms $g(Z)^{-1} d g(Z)$. Consider instead the local transformation

$$
g(Z) \rightarrow g\left(Z^{\prime}\right)=g(Z) e^{i v^{A}(Z) T_{A}} e^{-i \phi^{a b}(v, Z) J_{a b}},
$$

with $v^{A} T_{A}=v^{a} P_{a}+\bar{v}_{\alpha} Q^{\alpha}$. As noted earlier, the right action of the group on representative of cosets does not descend to a canonically defined transformation on $G / H$ : the transformation $Z \rightarrow Z^{\prime}(Z)$ determined by (6) is

\footnotetext{
${ }^{6}$ The term "action" appears twice in this sentence, once in the context of the integral of a Lagrangian, and once in the context of transformations on a group manifold generated by the group structure; in future use of this term, it should be clear from the context which of these is applicable.
} 
dependent on the choice of slice $g(Z)$. In infinitesimal form, the relationship between $v^{A}(Z)$ and $\delta Z=Z^{\prime}-Z$ is given by (8),

$$
v^{A}(Z)=\delta Z^{M} E_{M}^{A}(Z),
$$

where $E_{M}{ }^{A}(Z)$ is the vielbein on the coset superspace. Thus, setting $v^{a}(Z)=$ 0 corresponds to the condition

$$
0=\delta Z^{M} E_{M}^{a}(Z)
$$

This is one of the criteria which characterize a $\kappa$-transformation on the worldvolume of a p-brane embedded in a curved superspace [16, 17. It determines the transformation $\delta x^{m}$ of the even superspace coordinates of the embedded worldvolume in terms of $\delta \theta^{\alpha}$, the variation of the odd coordinates.

So the condition that $\delta Z^{M} E_{M}{ }^{a}(Z)$ vanishes is naturally implemented in terms of a local right action depending only on the fermionic generators of the supergroup[. As there is no canonically defined right action of $G$ on $G / H$, the implementation of a $\kappa$-transformation in the above fashion would seem to depend on the parameterization of the coset superspace. However, this is not true provided the right action is a local symmetry ; it is then a reflection of the presence of unphysical degrees of freedom which can be eliminated by a choice of gauge for the local symmetry.

The latter principle is well known in the standard construction of a sigma model (not necessarily supersymmetric) with a target space which is a coset space. Generically [26], one starts with an action of the form

$$
S=\int d x\left(g^{-1} \partial_{m} g\right)^{A}\left(g^{-1} \partial^{m} g\right)^{B} \delta_{A B}
$$

where $g(x)=\exp ^{i\left(\xi^{A}(x) T_{A}+\xi^{\bar{I}}(x) T_{\bar{I}}\right)} \in G$, with $T_{\bar{I}}$ the generators of $H$, and where $\delta_{A B}$ is a $H$-invariant metric. The sigma model possess a local symmetry under the right action of $H$ since $\left(g^{-1} \partial_{m} g\right)^{A}$ transforms linearly under such transformations (assuming the generators $T_{A}$ provide a representation of $H$ under commutation with the $T_{\bar{I}}$ ). This gauge symmetry can be fixed by setting $\xi^{\bar{I}}$ to zero, giving a parameterization of $G / H$ in a physical gauge in

\footnotetext{
${ }^{7}$ Of course, there are additional criteria which a $\kappa$-symmetry transformation must fulfill, as will be discussed shortly. In particular, only a subset of the supersymmetry generators is involved in a $\kappa$-symmetry transformation, and this subset depends on the point on the embedded worldvolume.
} 
terms of the slice $g(\xi)=e^{i \xi^{A} T_{A}}$. Since it is constructed from the Cartan form $g^{-1} d g$, the action $S$ also has a global symmetry under the left action of $G$. In particular, this global left action gives a linear realization of the subgroup $H$ on the physical degrees of freedom $\xi^{A}$, while the rest of the generators of $G$ are realized nonlinearly. Even in the physical gauge, there is still a symmetry under the local right action of $H$.

In the case of $p$-brane actions in coset superspaces $G / H$, a local symmetry under the right action of a Lorentz subgroup $H$ has been fixed in choosing a "slice" $g(Z)$ which is parameterized in the form $g(Z)=e^{i\left(x^{m} \delta_{m}{ }^{a} P_{a}+\bar{\theta} Q\right)}$. If the Wess-Zumino term is appropriately normalized, then the action possesses a $\kappa$-symmetry, which, as argued above, takes the form of a local right action generated by a subset of the supersymmetry generators. Although this right action is not well-defined on $G / H$, it suggests that the theory really describes degrees of freedom on a smaller coset space $G / \tilde{H}$, where $\tilde{H} \supset H$ includes an appropriate subset of the fermionic generators. In this case the right action is well-defined, independent of the parameterization of $G / H$ with which the construction starts. Further, under the left action of $G$, the fermionic generators in $\tilde{H}$ will be realized linearly on the physical degrees of freedom, while the remaining fermionic generators will still be nonlinearly realized. This is precisely the requirement for the effective action describing the low energy excitations of a BPS state which preserves only half the supersymmetry. It is this interpretation of $\kappa$-symmetry which we wish to pursue. It should be pointed out that the situation is actually more subtle, because the subgroup $\tilde{H}$ varies from point to point of the embedded worldvolume.

To illustrate the above, we review $p$-branes in flat superspace, which is a coset superspace $G / H$ with $G$ a super-Poincaré group and $H$ its Lorentz subgroup. The coset is parameterized in the form $g(Z)=e^{i\left(x^{a} P_{a}+\bar{\theta} Q\right)}$, and the Cartan form

$$
\begin{aligned}
g(Z)^{-1} d g(Z) & =i \Pi^{a} P_{a}+i d \bar{\theta} Q \\
& =i\left(d x^{a}-i\left(\bar{\theta} \Gamma^{a} d \theta\right)\right) P_{a}+i d \bar{\theta} Q
\end{aligned}
$$

provides a vielbein on flat superspace which is invariant under global supersymmetry transformations

$$
\delta x^{a}=i\left(\bar{\epsilon} \Gamma^{a} \theta\right), \quad \delta \theta=\epsilon .
$$

In infinitesimal form, the local right action $g(Z) \rightarrow g(Z) e^{i \bar{\epsilon} Q}$ induces the transformations

$$
\delta x^{a}=-i\left(\bar{\epsilon} \Gamma^{a} \theta\right), \quad \delta \theta=\epsilon
$$


(a compensating Lorentz transformation is not required in flat superspace). The vielbein transforms as

$$
\delta \Pi^{a}=-2 i\left(\bar{\epsilon} \Gamma^{a} d \theta\right), \quad \delta d \theta=d \epsilon .
$$

The Lorentz invariant metric $\eta_{a b}$ on the bosonic part of flat superspace can be pulled back to the $(p+1)$-dimensional worldvolume of the $p$-brane to yield a worldvolume metric

$$
G_{i j}=\frac{\partial Z^{M}}{\partial \sigma^{i}} \frac{\partial Z^{N}}{\partial \sigma^{j}} \Pi_{M}^{a} \Pi_{N}^{b} \eta_{a b} \equiv \Pi_{i}^{a} \Pi_{j}^{b} \eta_{a b}
$$

where $\sigma^{i}$ are coordinates on the worldvolume. The kinetic term $S_{0}$ in the $p$ brane action is a generalization of the massive spinning particle action, being proportional to the volume of the woldvolume with respect to the induced metric:

$$
S_{0}=\int d^{(p+1)} \sigma \sqrt{\operatorname{det} G} .
$$

Since $\Pi^{a}$ can be written in the form

$$
i \Pi^{a} P_{a}=g(x, \theta)^{-1} D g(x, \theta),
$$

where $D g=d g-i g(d \bar{\theta} Q)$ is a covariant derivative of $g, \Pi^{a}$ transforms linearly under the local right action $g(Z) \rightarrow g(Z) e^{i \bar{\epsilon} Q}$. However, $G_{i j}$ is not invariant under this transformation because $\eta_{a b}$ is an invariant metric only for the Lorentz subgroup of the super-Poincaré group, with the result that the variation of the kinetic term in the $p$-brane action is

$$
\delta \int d^{(p+1)} \sigma \sqrt{\operatorname{det} G}=-2 i \int d^{(p+1)} \sigma \sqrt{\operatorname{det} G}\left(\bar{\epsilon} \Gamma^{i} \partial_{i} \theta\right) .
$$

Here, the $\Gamma_{i}$ are worldvolume gamma matrices obtained by pulling back the spacetime gamma matrices,

$$
\Gamma_{i}=\frac{\partial Z^{M}}{\partial \sigma^{i}} \Pi_{M}^{a} \Gamma_{a}
$$

In the usual manner for the construction of sigma models with degrees of freedom on a coset space [26], if $\Pi_{i}^{a}$ and $\Pi_{j}{ }^{b}$ could be contracted with an invariant metric for the full super-Poincaré group, then an action could be constructed with a local fermionic symmetry. It would describe degrees of freedom living in the space of left cosets of flat superspace under the local 
fermionic transformation $g(Z) \rightarrow g(Z) e^{i \bar{\epsilon} Q}$. However, an appropriate invariant metric does not exist.

As is now well known, it is possible to obtain partial invariance with respect to the local right action. The matrix

$$
\Gamma=\frac{i^{\left[\frac{p+1}{2}\right]}}{(p+1) !} \epsilon^{i_{0} i_{1} \cdots i_{p}} \Gamma_{i_{0}} \Gamma_{i_{1}} \cdots \Gamma_{i_{p}}
$$

has the property $\Gamma^{2}=1$, and commutes with the worldvolume gamma matrices $\Gamma_{i}$ when $p$ is even and anticommutes with them when $p$ is odd. The generators $J_{i j}=-\frac{i}{8}\left[\Gamma_{i}, \Gamma_{j}\right]$ therefore yield a reducible representation of the worldvolume Lorentz group, and the projection operators $P_{ \pm}=\frac{1}{2}(1 \pm \Gamma)$ provide a decomposition of this reducible representation into two parts. If it is possible to find a contribution $S_{W Z}$ to the action such that under the local transformations

$$
\delta\left(S_{0}+S_{W Z}\right)=-4 i \int d^{(p+1)} \sigma \sqrt{\operatorname{det} G}\left(\bar{\epsilon} P_{-} \Gamma^{i} \partial_{i} \theta\right)
$$

then $S_{0}+S_{W Z}$ will be invariant under transformations $g(Z) \rightarrow g(Z) e^{i \bar{\epsilon} Q}$ with $\bar{\epsilon}=\bar{\kappa} P_{+}$. These are the $\kappa$-symmetry transformations, which allow half of the fermionic degrees of freedom to be gauged away.

A contribution $S_{W Z}$ to the $p$-brane action with the required transformation property under the local right action and which is invariant under global supersymmetry (or a global left action) exists only for flat superspaces in which the $(p+2)$-form

$$
h=i^{\left[\frac{p+1}{2}\right]} \frac{i}{p !} \Pi^{a_{1}} \wedge \Pi^{a_{2}} \wedge \cdots \wedge \Pi^{a_{p}}\left(d \bar{\theta} \Gamma_{a_{1}} \Gamma_{a_{2}} \cdots \Gamma_{a_{p}} d \theta\right)
$$

is closed; this leads to the "brane-scan" restricting the relationship between the dimension of the worldvolume and the dimension of the spacetime in which it is embedded [18]. The closure of this form is equivalent to the integrability of

$$
\delta S_{W Z}=2 i \int d^{(p+1)} \sigma \sqrt{\operatorname{det} G}\left(\bar{\kappa} \Gamma \Gamma^{i} \partial_{i} \theta\right),
$$

\footnotetext{
${ }^{8}$ We use the convention $d \sigma^{i_{0}} \wedge d \sigma^{i_{1}} \wedge \cdots d \sigma^{i_{p}}=d^{(p+1)} \sigma \sqrt{\operatorname{det} G} \epsilon^{i_{0} i_{1} \cdots i_{p}}$. Also, a rotation to a Euclidean metric has been made for convenience.
} 
in the sense that only in these cases is there a local quantity $S_{W Z}$ whose variation yields the right hand side of (16). This is similar to the WessZumino consistency condition for anomalies [27].

In this case, the $p$-brane action is

$$
S_{0}+S_{W Z}=\int d^{(p+1)} \sigma \sqrt{\operatorname{det} G}+\int \phi^{*} b,
$$

$\phi$ denotes the embedding of the worldvolume into superspace, and $b$ is a $(p+1)$-form defined by $d b=h$. To see how this expression for the WessZumino term arises, note that $\delta S_{W Z}$ can be written in the form

$$
\begin{aligned}
\delta S_{W Z} & =i^{\left[\frac{p+1}{2}\right]} \frac{2 i}{p !} \int d \sigma^{i_{1}} \wedge \cdots \wedge d \sigma^{i_{p+1}}\left(\bar{\kappa} \Gamma_{i_{1}} \cdots \Gamma_{i_{p}} \partial_{i_{p+1}} \theta\right) \\
& =i^{\left[\frac{p+1}{2}\right]} \frac{2 i}{p !} \int \phi^{*} \Pi^{a_{1}} \wedge \cdots \wedge \Pi^{a_{p}} \wedge\left(\bar{\kappa} \Gamma_{a_{1}} \cdots \Gamma_{a_{p}} d \theta\right) .
\end{aligned}
$$

If we consider a path $g(t)=e^{i\left(x^{a} P_{a}+t \bar{\theta} Q\right)}$ in the fermionic directions on the coset superspace, then this is equivalent to

$$
\frac{d}{d t} S_{W Z}(t)=i^{\left[\frac{p+1}{2}\right]} \frac{2 i}{p !} \int \phi^{*} \Pi^{a_{1}}(t) \wedge \cdots \wedge \Pi^{a_{p}}(t) \wedge\left(\bar{\nu}(t) \Gamma_{a_{1}} \cdots \Gamma_{a_{p}} t d \theta\right)
$$

where

$$
g(t)^{-1} d g(t)=i \Pi^{a}(t) P_{a}+i t d \bar{\theta} Q=i\left(d x^{a}-i t^{2}\left(\bar{\theta} \Gamma_{a} d \theta\right)\right) P_{a}+i t d \bar{\theta} Q
$$

and $g(t)^{-1} \frac{d}{d t} g(t)=i(\bar{\nu}(t) Q)$. The reason that a derivative with respect to $t$ can be considered as an infinitesimal right action with parameter $\bar{\nu}(t)_{\alpha}$ is that $\frac{d}{d t} g(t)$ can be written in the form $g(t) \cdot\left(g(t)^{-1} \frac{d}{d t} g(t)\right)$, mimicing an infinitesimal local right action. Equation (17) can be integrated to yield

$$
S_{W Z}=i^{\left[\frac{p+1}{2}\right]} \frac{2 i}{p !} \int_{0}^{1} d t \int \phi^{*} \Pi^{a_{1}}(t) \wedge \cdots \wedge \Pi^{a_{p}}(t) \wedge\left(\bar{\nu}(t) \Gamma_{a_{1}} \cdots \Gamma_{a_{p}} t d \theta\right) .
$$

\footnotetext{
${ }^{9}$ Note that there is no $P_{a}$ term in $g(t)^{-1} \frac{d}{d t} g(t)$ for the particular choice of path $g(t)$ given because $d t\left(\bar{\theta} \Gamma^{a} \theta\right)=0$; for a more general path $\theta(t)$, there would be such terms, in which case it would also be necesary to include an integration of the variation of $S_{W Z}$ under the bosonic part of the infinitesimal right action.

${ }^{10}$ The approach of integrating the variation of the Wess-Zumino term was used by Aganagic et al to construct D-p-brane Wess-Zumino terms in [28].
} 
The $t$ integral on right hand side of this expression is the standard cohomological construction of a form $b$ with the property $d b=h$ in the case where $h=i^{\left[\frac{p+1}{2}\right]} \frac{i}{p !} \Pi^{a_{1}} \wedge \Pi^{a_{2}} \wedge \cdots \wedge \Pi^{a_{p}}\left(d \bar{\theta} \Gamma_{a_{1}} \Gamma_{a_{2}} \cdots \Gamma_{a_{p}} d \theta\right)$ is closed.

Note that although flat superspace can be constructed as a coset space of the super-Poincaré group by the Lorentz group, it is in fact itself a group, the super-translation group (as emphasized in [7]). So the left and right actions of the super-translation group on flat superspace are well-defined. This is reflected in the fact that no compensating Lorentz transformation is required in the definition of the right action of the super-Poincaré group on $g(Z)=e^{i\left(x^{a} P_{a}+\bar{\theta} Q\right)}$. The entire discussion of $p$-branes in flat superspace above can therefore be carried out without reference to cosets. Nevertheless, it is useful to consider flat superspace as a coset space, because it makes obvious the origin of the local Lorentz invariance of the $p$-brane action, and also because it points to the generalizations required for coset superspaces $G / H$ which are not groups and for which there is no naturally defined right action of $G$.

\section{Incorporating Worldvolume Diffeomorphisms}

Returning to the case of $p$-brane actions in general coset superspace backgrounds, they are by construction also invariant under worldvolume diffeomorphisms. This ensures that there are only physical Goldstone modes corresponding to the generators $P_{a}$ of superspace translations which are broken by the embedding of the $p$-brane into superspace. The unphysical bosonic Goldstone degrees of freedom in the covariant Green-Schwarz action can be eliminated by a choice of gauge for the worldvolume diffeomorphisms. Since symmetry with respect to the worldvolume diffeomorphisms performs the same role as the $\kappa$-symmetry transformations, namely the elimination of unphysical Goldstone degrees of freedom, then if the above interpretation of the $\kappa$-symmetry transformations as enlarging the isotropy group in the construction of the coset superspace is correct, the worldvolume diffeomorphisms should also have an interpretation in terms of the local right action of a subgroup of the supergroup $G$ on the coset superspace $G / H$. This is indeed the case, as we now demonstrate.

If $Z^{M}(\sigma)$ denotes the embedding of the $(p+1)$-dimensional worldvolume (with coordinates $\sigma^{i}$ ) of a $p$-brane into the coset superspace, then since the spacetime coordinates are worldvolume scalars, they transform under 
an infinitesimal worldvolume diffeomorphism generated by the worldvolume vector field $v^{i}(\sigma) \frac{\partial}{\partial \sigma^{i}}$ as

$$
\delta_{v} Z^{M}(\sigma)=v^{i}(\sigma) \frac{\partial Z^{M}(\sigma)}{\partial \sigma^{i}} .
$$

Using (8) and (9), such a transformation of the spacetime coordinates is induced by a right action of the form

$$
g(Z(\sigma)) \rightarrow g\left(Z^{\prime}(\sigma)\right)=g(Z(\sigma)) e^{i v^{A}(\sigma) T_{A}+i w^{a b}(\sigma) J_{a b}} e^{-i \phi^{a b}(\sigma) J_{a b}}
$$

with

$$
v^{A}(\sigma)=v^{i}(\sigma) \frac{\partial Z^{M}(\sigma)}{\partial \sigma^{i}} E_{M}^{A}(Z(\sigma))
$$

and

$$
w^{a b}(\sigma)=v^{i}(\sigma) \frac{\partial Z^{M}(\sigma)}{\partial \sigma^{i}} \Omega_{M}^{a b}(Z(\sigma))-\phi^{a b}(v, \sigma) .
$$

It is possible to choose $w^{a b}(\sigma)$ so that the compensating transformation vanishes, namely $w^{a b}(\sigma)=v^{i}(\sigma) \frac{\partial Z^{M}(\sigma)}{\partial \sigma^{i}} \Omega_{M}{ }^{a b}(Z(\sigma))$. In this case the diffeomorphism is generated in the form

$$
g\left(Z^{\prime}(\sigma)\right)=g(Z(\sigma)) e^{i v^{i}(\sigma)\left(E_{i}{ }^{A}(\sigma) T_{A}+\Omega_{i}^{a b}(\sigma) J_{a b}\right)},
$$

where $E_{i}{ }^{A}=\frac{\partial Z^{M}}{\partial \sigma^{i}} E_{M}{ }^{A}$ and $\Omega_{i}{ }^{a b}=\frac{\partial Z^{M}}{\partial \sigma^{i}} \Omega_{M}{ }^{a b}$ are the pullbacks of the vielbein and connection to the worldvolume. This is very similar to the implementation of diffeomorphisms in superspaces in terms of so-called supergauge transformations 29].

To check this, computing $g\left(Z^{\prime}\right)^{-1} \frac{\partial}{\partial \sigma^{i}} g\left(Z^{\prime}\right)$ with $v^{i}(\sigma)$ infinitesimal yields (upon use of the expressions (4) for the torsion and curvature of a coset superspace) the correct transformations

$$
\begin{aligned}
\delta_{v} E_{i}{ }^{A}(\sigma) & =\frac{\partial v^{j}(\sigma)}{\partial \sigma^{i}} E_{j}{ }^{A}(\sigma)+v^{j}(\sigma)\left(\partial_{j} E_{i}{ }^{A}(\sigma)\right) \\
\delta_{v} \Omega_{i}{ }^{a b}(\sigma) & =\frac{\partial v^{j}(\sigma)}{\partial \sigma^{i}} \Omega_{j}{ }^{a b}(\sigma)+v^{j}(\sigma)\left(\partial_{j} \Omega_{i}{ }^{a b}(\sigma)\right)
\end{aligned}
$$

for the pullbacks of the vielbein and connection. 


\section{The Algebra of $\kappa$-Transformations and Dif- feomorphisms}

Both the $\kappa$-symmetry transformations and the diffeomorphisms are local symmetries which remove unphysical Goldstone degrees of freedom from the covariant Green-Schwarz action for a $p$-brane propagating in a coset superspace $G / H$, leaving physical Goldstone modes only for those generators of the supergroup $G$ which are broken by the embedding of the $p$-brane in superspace. In the left action of $G$ on $G / H$, the broken generators are realized nonlinearly on the physical degrees of freedom, while the unbroken generators are realized linearly. As already mentioned, this is naturally achieved if the $\kappa$-symmetry transformations and the diffeomorphisms arise from a local right action of special linear combinations of the generators of the supergroup $G$ on the coset superspace representatives $g(Z(\sigma))$; however, for this picture to be consistent, these linear combinations of generators must close under commutation and so generate a subgroup of the supergroup. This will then have the effect of enlarging the isotropy group of the coset superspace to a subgroup $\tilde{H}$ which contains these linear combinations of generators in addition to the Lorentz generators. The subgroup $\tilde{H}$ varies from point to point on the embedded worldvolume.

It has already been demonstrated in a general context that both the $\kappa$ symmetry transformations and the diffeomorphisms can indeed be realized in terms of a local right action. It thus remains to show that the corresponding generators form a subalgebra of the full superalgebra $G$. In other words, it is necessary to show that the generators of $\kappa$-symmetry transformations, worldvolume diffeomorphisms and Lorentz transformations form a closed algebra under commutation if the above interpretation of these transformations as local right actions is to be consistent.

Before calculating the appropriate commutators, the form of $\kappa$-symmetry transformations in a general coset superspace must be specified. As in flat superspace, they involve the pullback to the worldvolume of various structures on the coset superspace. These include the worldvolume metric, which is the pullback of the Lorentz invariant metric $\eta_{a b}$ :

$$
G_{i j}(\sigma)=E_{i}^{a}(Z(\sigma)) E_{j}^{b}(Z(\sigma)) \eta_{a b}
$$

where $E_{i}{ }^{A}=\frac{\partial Z^{M}}{\partial \sigma^{i}} E_{M}{ }^{A}$. Similarly, worldvolume gamma matrices are obtained 
by pulling back those defined in spacetime relative to the metric $\eta_{a b}$ :

$$
\Gamma_{i}(\sigma)=E_{i}^{a}(Z(\sigma)) \Gamma_{a}
$$

satisfies $\left\{\Gamma_{i}(\sigma), \Gamma_{j}(\sigma)\right\}=2 G_{i j}(\sigma)$. The matrix

$$
\Gamma=\frac{i^{\left[\frac{p+1}{2}\right]}}{(p+1) !} \epsilon^{i_{0} i_{1} \cdots i_{p}} \Gamma_{i_{0}} \Gamma_{i_{1}} \cdots \Gamma_{i_{p}}
$$

has the same properties as in the case of flat superspace, and the projection operators $P_{ \pm}=\frac{1}{2}(1 \pm \Gamma)$ provide a decomposition of the spinor representation of the worldvolume Lorentz group provided by the worldvolume gamma matrices into two parts. A related projection operator is the symmetric tensor $K^{a b}(\sigma)=E_{i}{ }^{a}(\sigma) G^{i j}(\sigma) E_{j}{ }^{b}(\sigma)$, satisfying $K_{a}{ }^{b} K_{b}{ }^{c}=K_{a}{ }^{c}$. It has the property

$$
E_{i}^{b}(\sigma) K_{b}{ }^{a}(\sigma)=E_{i}{ }^{a}(\sigma) .
$$

If $\phi=E^{a} \phi_{a}$ is a bosonic one-form on the coset superspace, then $K_{a}{ }^{b}(\sigma) \phi_{b}(\sigma)$ is the piece of $\phi_{a}$ which pulls back to the worldvolume, while $(1-K)_{a}^{b}(\sigma) \phi_{b}(\sigma)$ has a vanishing pull-back. For the spacetime gamma matrices $\Gamma_{a}$, this means that $\Gamma_{b} K_{b}{ }^{a}(\sigma)$ pulls back to a linear combination of the worldvolume gamma matrices, $\Gamma^{b} K_{b}{ }^{a}(\sigma)=\Gamma^{i}(\sigma) E_{i}^{a}(\sigma)$, while $\Gamma^{b}(1-K)_{b}{ }^{a}(\sigma)$ consists of the gamma matrices which are "transverse" to the worldvolume gamma matrices (and therefore anticommutes with all of them). This will be important in what follows.

The $\kappa$-symmetry transformations for a $p$-brane embedded in flat superspace are of the form

$$
g(Z(\sigma)) \rightarrow g(Z(\sigma)) e^{i \bar{\kappa}(\sigma) P_{+}(\sigma) Q} .
$$

Note that the transformation refers to a particular point $Z(\sigma)$ on the embedded worldvolume, and that the spinor $\kappa^{\alpha}$ is an arbitrary function of $\sigma$. In a curved coset superspace, the point $g(Z(\sigma)) e^{i \bar{\kappa}(\sigma) P_{+}(\sigma) Q}$ no longer lies on the slice $g(Z)=e^{i Z^{M} \delta_{M}^{A} T_{A}}$, and so a compensating Lorentz transformation is necessary if $\kappa$-symmetry transformations are to take this form. Alternatively, by the same method used in the case of diffeomorphisms, it is possible to implement the $\kappa$-symmetry transformation in such a manner that a compensating Lorentz transformation is not necessary, in which case the right action is of the form

$$
g(Z(\sigma)) \rightarrow g\left(Z^{\prime}(\sigma)\right)=g(Z(\sigma)) e^{i \bar{\kappa}(\sigma) P_{+}(\sigma) Q+i\left(\bar{\kappa} P_{+} C^{-1}\right)^{\alpha} E_{\alpha}{ }^{N} \Omega_{N}^{a b}(\sigma) J_{a b}} .
$$


In deriving this, it is necessary to use

$$
\delta_{\kappa} Z^{M} \Omega_{M}^{a b}=\delta_{\kappa} Z^{M} E_{M}{ }^{A} E_{A}{ }^{N} \Omega_{N}{ }^{a b},
$$

where $E_{A}^{N}$ is the inverse vielbein, as well as the fact that $\delta_{\kappa} Z^{M} E_{M}^{\alpha}=$ $\left(\bar{\kappa} P_{+} C^{-1}\right)^{\alpha}$.

In the appendix, it is shown that for infinitesimal worldvolume diffeomorphisms and $\kappa$-symmetry transformations, defined in terms of the right actions (18) and (21), the following commutation relations hold:

$$
\begin{aligned}
{\left[\delta_{v_{1}}, \delta_{v_{2}}\right] } & =\delta_{v_{3}} \\
{\left[\delta_{v}, \delta_{\kappa}\right] } & =\delta_{\kappa^{\prime}}
\end{aligned}
$$

with $v_{3}^{i}=v_{2}^{j}\left(\partial_{j} v_{1}^{i}\right)-v_{1}^{j}\left(\partial_{j} v_{2}^{i}\right)$ and $\kappa^{\prime}=-v^{i} \partial_{i} \kappa$. The first commutator yields the expected algebra for worldvolume diffeomorphisms, and the second commutator is consistent with long-established properties of the algebra of $\kappa$ symmetry transformations [1, 23].

The commutation relations (22) are valid independent of the dimension of the spacetime formed by the coset superspace into which the $p$-brane is embedded. However, this is not true for the commutator $\left[\delta_{\kappa_{1}}, \delta_{\kappa_{2}}\right]$ of a pair of $\kappa$-symmetry transformations. As shown in the appendix, such a commutator yields a linear combination of a diffeomorphism (parameterized by the worldvolume vector field $\left.v^{i}=-2 i\left(\bar{\kappa}_{2} P_{+} \Gamma^{i} P_{ \pm} \kappa_{1}\right)\right)$, a Lorentz transformation and another $\kappa$-symmetry transformation $\amalg$ if the following conditions are satisfied:

(i) The dimension of the spacetime into which the $p$-brane is embedded is such that the gamma matrix identities

$$
0=\left(C \Gamma_{a}\right)_{\alpha(\beta}\left(C \Gamma^{a}\right)_{\gamma \delta)}
$$

for $p=1$ and

$$
0=\left(C \Gamma_{a_{1}}\right)_{(\alpha \beta}\left(C \Gamma^{a_{1} a_{2} \cdots a_{p}}\right)_{\gamma \delta)}
$$

for $p>1$ are true (the round brackets on spinor indices denote symmetrization). This yields the usual "brane scan" for $p$-branes whose bosonic degrees of freedom are worldsheet scalars [18].

\footnotetext{
${ }^{11}$ The calculation in the appendix does not give the explicit form of the Lorentz transformation and the $\kappa$-symmetry transformation, although these could be extracted with a little more effort. Also note that conditions (i) and (ii) are only shown to be sufficient rather than necessary conditions for the algebra to close.
} 
(ii) For $p>1$, the additional condition $0=\left(P_{-} \Gamma^{i}\right)^{\alpha}{ }_{\beta} E_{i}{ }^{\beta}$ must also be satisfied.

Condition (ii) is one of the equations of motion for a $p$-brane, namely that which follows from

$$
\delta S=\int d^{(p+1)} \sigma \sqrt{\operatorname{det} G}\left(\delta Z^{M}\right) E_{M}^{\alpha}\left(C P_{-} \Gamma^{i}\right)_{\alpha \beta} E_{i}^{\alpha}
$$

under a variation $\left(\delta Z^{M}\right) E_{M}^{\alpha}$ of the $p$-brane action $S$. This is consistent with the existence of a $\kappa$-symmetry form $\left(\delta_{\kappa} Z^{M}\right) E_{M}{ }^{\alpha}=\left(\bar{\kappa} P_{+} C^{-1}\right)^{\alpha}$. As discussed in the case of flat superspace, (23) can be taken as a definition of the $p$-brane action [28], in that integrating this gives the usual $p$-brane action (with the kinetic term coming from the piece in $P_{-}$proportional to 1 and the WessZumino term coming from the piece in $P_{-}$proportional to $\Gamma$ ). So except for $p=1$ (a string), the algebra of diffeomorphisms and $\kappa$-symmetry transformations closes only on-shell. It is interesting that requiring the algebra of the $\kappa$-symmetry transformations, worldvolume diffeomorphisms and Lorentz transformations to close on itself yields a condition which can be used to construct the $p$-brane action.

\section{Conclusion}

The suggestion [12, 14, 15] that $\kappa$-symmetry transformations are related to the right action of the supergroup $G$ on a coset superspace $G / H$ has been examined for a special class of coset superspaces which are currently of interest in studies of $p$-branes propagating in curved spacetimes. It has been shown that the condition

$$
\delta Z^{M} E_{M}^{a}=0
$$

which is accepted as one of the hallmarks of a $\kappa$-symmetry transformation for $p$-branes embedded in curved superspaces [16, 17] arises very naturally if the $\kappa$-symmetry transformation is implemented in terms of a right action of the supergroup $G$ on $G / H$. Further, a physical interpretation of this right action in terms of an enlargement of the isotropy subgroup from the Lorentz subgroup of $G$ has been suggested. This is consistent with the role of both worldvolume diffeomorphisms and $\kappa$-symmetry transformations in covariant Green-Schwarz p-brane actions, namely in the elimination of unphysical Goldstone degrees of freedom. Requiring that the algebra of worldvolume diffeomorphisms, $\kappa$-symmetry transformations and Lorentz transformations 
closes on itself at each point of the embedded worldvolume leads to the usual "brane scan" for $p$-branes with worldvolume degrees of freedom which are scalars $18 \|$. In the case $p>1$, closure of the algebra also requires that one of the usual equations of motion for a $p$-brane be true. It was pointed out that this condition can in fact be used to reconstruct the $p$-brane action.

It is interesting to speculate on the relationship of the approach in this paper to the original formulation of the "brane scane" in terms of the existence of suitable closed differential forms in the embedding superspace from which the Wess-Zumino term in the covariant Green-Schwarz $p$-brane action can be constructed [18, 7]. The closure of the algebra of worldvolume diffeomorphisms, $\kappa$-symmetry transformations and Lorentz transformations corresponds to an integrability condition on certain vector fields, and this usually has a dual formulation in terms of differential forms. The results of this paper are presumably also closely related to the geometric interpretation of $\kappa$-symmetry transformations in terms of superembeddings [10, 9, 11.

Acknowledgement: I wish to thank Professor P. Townsend for pointing out reference [14].

\section{Appendix}

In this appendix, the conditions under which the generators of diffeomorphisms and $\kappa$-symmetry transformations form a closed algebra under commutation are examined.

Consider first the diffeomorphisms, generated by the right action (18). The generator of diffeomorphisms depends on the point $Z(\sigma)$ on the embedded worldvolume through the presence of $E_{i}{ }^{A}(Z(\sigma))$ and $\Omega_{i}{ }^{a b}(Z(\sigma)$ ) (the latter vanishes in flat superspace). Thus, if a pair of diffeomorphisms are successively applied, the generator of the second diffoemorphism depends on the point $Z^{\prime}$ resulting from the first diffeomorphism:

$$
g(Z) \rightarrow g(Z) e^{i v_{1}^{i}(\sigma)\left(E_{i}^{A}(Z) T_{A}+\Omega_{i}^{a b}(Z) J_{a b}\right)} e^{i v_{2}^{j}(\sigma)\left(E_{j}^{B}\left(Z^{\prime}\right) T_{B}+\Omega_{j}{ }^{c d}\left(Z^{\prime}\right) J_{c d}\right)} .
$$

For infinitesimal diffeomorphisms, the arguments of the exponentials can be combined as

$$
i\left(v_{1}+v_{2}\right)^{i}\left(E_{i}^{A} T_{A}+\Omega_{i}^{a b} J_{a b}\right)
$$




$$
\begin{aligned}
& +\frac{1}{2}\left[i v_{1}^{i}\left(E_{i}{ }^{A} T_{A}+\Omega_{i}{ }^{a b} J_{a b}\right), i v_{2}^{j}\left(E_{j}{ }^{B} T_{B}+\Omega_{j}{ }^{c d} J_{c d}\right)\right] \\
& +i v_{2}^{j}\left(\delta_{v_{1}} E_{j}{ }^{B} T_{B}+\delta_{v_{1}} \Omega_{j}{ }^{c d} J_{c d}\right) .
\end{aligned}
$$

If the diffeomorphisms are performed in reverse order, the same expression with 1 and 2 interchanged results. The commutator of a pair of diffeomorphisms is the difference of these expressions. Using the result (19) and the expression (四) for the torsion and curvature of the coset superspace, one finds

$$
\left[\delta_{v_{1}}, \delta_{v_{2}}\right]=\delta_{v_{3}}
$$

with $v_{3}^{i}=v_{2}^{j}\left(\partial_{j} v_{1}^{i}\right)-v_{1}^{j}\left(\partial_{j} v_{2}^{i}\right)$.

In checking the commutator of a diffeomorphism and a $\kappa$-symmetry transformation, the variations of the pulled back vielbein and connection are required:

$$
\begin{aligned}
\delta_{\kappa} E_{i}{ }^{a}= & -i E_{i}{ }^{\beta}\left(\bar{\kappa} P_{+} C^{-1}\right)^{\alpha} i f_{\alpha \beta}{ }^{a}-i\left(\bar{\kappa} P_{+} C^{-1} E^{M}\right) \Omega_{M}{ }^{c d} E_{i}{ }^{b} i f_{c d b}{ }^{a} \\
\delta_{\kappa} E_{i}{ }^{\alpha}= & \partial_{i}\left(\bar{\kappa} P_{+} C^{-1}\right)^{\alpha}+i\left(\bar{\kappa} P_{+} C^{-1}\right)^{\beta} E_{i}{ }^{a} i f_{a \beta}{ }^{\alpha} \\
& +i\left(\bar{\kappa} P_{+} C^{-1}\right)^{\beta} \Omega_{i}{ }^{a b} i f_{a b \beta}{ }^{\alpha}-i\left(\bar{\kappa} P_{+} C^{-1} E^{M}\right) \Omega_{M}{ }^{a b} E_{i}{ }^{\beta} i f_{a b \beta}{ }^{\alpha} \\
\delta_{\kappa} \Omega_{i}{ }^{a b}= & \partial_{i}\left(\left(\bar{\kappa} P_{+} C^{-1} E^{M}\right) \Omega_{M}{ }^{a b}\right)-i E_{i}{ }^{\beta}\left(\bar{\kappa} P_{+} C^{-1}\right)^{\alpha} i f_{\alpha \beta}{ }^{a b} \\
& -i\left(\bar{\kappa} P_{+} C^{-1} E^{M}\right) \Omega_{M}{ }^{c d} \Omega_{i}{ }^{e f} i f_{c d e f}{ }^{a b},
\end{aligned}
$$

where $\left(\bar{\kappa} P_{+} C^{-1} E^{M}\right)=\left(\bar{\kappa} P_{+} C^{-1}\right)^{\alpha} E_{\alpha}{ }^{M}$, and where all transformations are evaluated at a point $Z(\sigma)$ on the the embedded worldvolume. The results (24) are obtained by computing $g\left(Z^{\prime}\right)^{-1} \frac{\partial}{\partial \sigma^{2}} g\left(Z^{\prime}\right)$ with $g\left(Z^{\prime}\right)$ given by (21). Also required is the $\kappa$-symmetry transformation of the projection operator $P_{+}$, which is

$$
\delta_{\kappa} P_{+}=\frac{1}{2} \delta_{\kappa} \Gamma=\frac{1}{2}\left(\delta_{\kappa} E_{i}^{\bar{a}}\right) \Gamma_{\bar{a}} \Gamma^{i} \Gamma .
$$

Here, $\Gamma_{\bar{a}}$ is shorthand for $(1-K)_{a}{ }^{b} \Gamma_{b}$, and a sum over $\bar{a}$ is thus a restriction to a sum over the spacetime vector degrees of freedom which are transverse to the worldvolume. In computing $\delta_{\kappa} P_{+}$, it is necessary to use the fact that $\Gamma^{b} K_{b}{ }^{a}=\Gamma^{i} E_{i}{ }^{a}$ anticommutes with $\Gamma^{c}(1-K)_{c}{ }^{d}$, and that $\sqrt{\operatorname{det} G} \epsilon^{i_{0} i_{1} \cdots i_{p}}$ transforms as a scalar.

As with the commutator of a pair of diffeomorphisms, in computing the commutator of a diffeomorphism and a $\kappa$-symmetry transformation, the second transformation acts at a transformed point, and one finds

$$
\left[\delta_{v}, \delta_{\kappa}\right]=-\left[v^{i}\left(E_{i}{ }^{A} T_{A}+\Omega_{i}{ }^{a b} J_{a b}\right),\left(\bar{\kappa} P_{+} Q\right)+\left(\bar{\kappa} P_{+} C^{-1} E^{M}\right) \Omega_{M}{ }^{a b} J_{a b}\right]
$$




$$
\begin{aligned}
& -i v^{i}\left(\delta_{\kappa} E_{i}{ }^{A}\right) T_{A}-i v^{i}\left(\delta_{\kappa} \Omega_{i}^{a b}\right) J_{a b} \\
& +i \bar{\kappa}\left(\delta_{v} P_{+}\right) Q+i \bar{\kappa} \delta_{v}\left(P_{+} C^{-1} E^{M} \Omega_{M}^{a b}\right) J_{a b} .
\end{aligned}
$$

By computing $g\left(Z^{\prime}\right) \partial_{i} g\left(Z^{\prime}\right)$ with $g\left(Z^{\prime}\right)$ given by (21), the first three terms in this expression are easily shown to be

$$
-i \partial_{i}\left(\bar{\kappa} P_{+}\right) Q-i \partial_{i}\left(\left(\bar{\kappa} P_{+} C^{-1} E^{M}\right) \Omega_{M}^{a b}\right) J_{a b}
$$

Combining this with the fact that $\delta_{v} \phi=v^{i} \partial_{i} \phi$ for worldvolume scalars yields

$$
\left[\delta_{v}, \delta_{\kappa}\right]=\delta_{\kappa^{\prime}}
$$

with $\kappa^{\prime}=-v^{i} \partial_{i} \kappa$.

The only difficult step in computing the algebra of right transformations involves a pair of $\kappa$-symmetry transformations. This involves

$$
\begin{aligned}
{\left[\delta_{\kappa_{1}}, \delta_{\kappa_{2}}\right]=} & {\left[i \overline{\kappa_{1}} P_{+} Q+i\left(\bar{\kappa}_{1} P_{+} C^{-1} E^{M}\right) \Omega_{M}^{a b} J_{a b}, i \overline{\kappa_{2}} P_{+} Q\right.} \\
& \left.+i\left(\bar{\kappa}_{2} P_{+} C^{-1} E^{N}\right) \Omega_{N}{ }^{c d} J_{c d}\right]+i \overline{\kappa_{2}}\left(\delta_{\kappa_{1}} P_{+}\right) Q \\
& +i \overline{\kappa_{2}} \delta_{\kappa_{1}}\left(P_{+} C^{-1} E^{M} \Omega_{M}^{a b}\right) J_{a b}-i \overline{\kappa_{1}}\left(\delta_{\kappa_{2}} P_{+}\right) Q \\
& -i \overline{\kappa_{1}} \delta_{\kappa_{2}}\left(P_{+} C^{-1} E^{M} \Omega_{M}^{a b}\right) J_{a b} .
\end{aligned}
$$

Computing the commutator and collecting all the terms proportional to $J_{a b}$ in a Lorentz transformation $\delta_{L}$,

$$
\begin{aligned}
{\left[\delta_{\kappa_{1}}, \delta_{\kappa_{2}}\right]=} & 2\left(\bar{\kappa}_{2} P_{+} \Gamma^{a} P_{ \pm} \kappa_{1}\right) P_{a} \\
& +\left(\bar{\kappa}_{2} P_{+} C^{-1} E^{M}\right) \Omega_{M}{ }^{a b}\left(\bar{\kappa}_{1} P_{+} C^{-1}\right)^{\alpha} i f_{a b \alpha}{ }^{\beta} Q_{\beta} \\
& -\left(\bar{\kappa}_{1} P_{+} C^{-1} E^{M}\right) \Omega_{M}{ }^{a b}\left(\bar{\kappa}_{2} P_{+} C^{-1}\right)^{\alpha} i f_{a b \alpha}{ }^{\beta} Q_{\beta} \\
& +\frac{i}{2} \bar{\kappa}_{2}\left(\delta_{\kappa_{1}} \Gamma\right) Q-\frac{i}{2} \bar{\kappa}_{1}\left(\delta_{\kappa_{2}} \Gamma\right) Q+\delta_{L},
\end{aligned}
$$

where the \pm in the first term applies to $p$ even/odd, and we have used $\left(C P_{+}\right)^{T}=-C P_{ \pm}$for $P$ even/odd. In fact,

$$
\Gamma^{b}(1-K)_{b}{ }^{a} P_{ \pm}=P_{-} \Gamma^{b}(1-K)_{b}^{a}
$$

so the first term becomes

$$
2\left(\bar{\kappa}_{2} P_{+} \Gamma^{i} P_{ \pm} \kappa_{1}\right) E_{i}^{a} P_{a}
$$


which is part of the generator of a diffeomorphism with parameter $v^{i}=$ $-2 i\left(\bar{\kappa}_{2} P_{+} \Gamma^{i} P_{ \pm} \kappa_{1}\right)$. Completing the generator of the diffeomorphism,

$$
\begin{aligned}
{\left[\delta_{\kappa_{1}}, \delta_{\kappa_{2}}\right]=} & \delta_{v}-2\left(\bar{\kappa}_{2} P_{+} \Gamma^{i} P_{ \pm} \kappa_{1}\right) E_{i}{ }^{\alpha} Q_{\alpha} \\
& +\left(\bar{\kappa}_{2} P_{+} C^{-1} E^{M}\right) \Omega_{M}{ }^{a b}\left(\bar{\kappa}_{1} P_{+} C^{-1}\right)^{\alpha} i f_{a b \alpha}{ }^{\beta} Q_{\beta} \\
& -\left(\bar{\kappa}_{1} P_{+} C^{-1} E^{M}\right) \Omega_{M}{ }^{a b}\left(\bar{\kappa}_{2} P_{+} C^{-1}\right)^{\alpha} i f_{a b \alpha}{ }^{\beta} Q_{\beta} \\
& +\frac{i}{2} \bar{\kappa}_{2}\left(\delta_{\kappa_{1}} \Gamma\right) Q-\frac{i}{2} \bar{\kappa}_{1}\left(\delta_{\kappa_{2}} \Gamma\right) Q+\delta_{L} .
\end{aligned}
$$

The third and fourth terms on the right-hand side cancel against the $\Omega_{M}{ }^{a b}$ dependent pieces of the last two terms, which are obtained from (24) and (25). To show that the remaining $Q$ dependent terms constitute a $\kappa$-symmetry transformation, it is only necessary to show that the pieces proportional to $P_{-} Q$ vanish, as a $\kappa$-symmetry transformation is generated by $i \bar{\kappa} P_{+} Q$ (plus a piece which can be absorbed into a Lorentz transformation) and so only involves $P_{+} Q$. Thus it suffices to prove

$$
\begin{aligned}
0= & -2\left(\bar{\kappa}_{2} P_{+} \Gamma^{i} P_{ \pm} \kappa_{1}\right)\left(\bar{E}_{i} P_{-} Q\right)+\left(\bar{\kappa}_{1} P_{+} \Gamma_{\bar{a}} E_{i}\right)\left(\bar{\kappa}_{2} \Gamma^{\bar{a}} \Gamma^{i} \Gamma P_{-} Q\right) \\
& -\left(\bar{\kappa}_{2} P_{+} \Gamma_{\bar{a}} E_{i}\right)\left(\bar{\kappa}_{1} \Gamma^{\bar{a}} \Gamma^{i} \Gamma P_{-} Q\right) .
\end{aligned}
$$

The sums over the restricted range $\bar{a}$ in the last terms (i.e. over spacetime vector indices transverse to the worldvolume, see after equation (25)) can be replaced by unrestricted sums by inclusion of appropriate projection operators, and the required identity is

$$
\begin{aligned}
0= & -2\left(\overline{\kappa_{2}^{ \pm}} \Gamma^{i} \kappa_{1}^{ \pm}\right)\left(\overline{E_{i}^{\mp}} Q^{-}\right)+\left(\overline{\kappa_{1}^{ \pm}} \Gamma_{a} E_{i}^{\mp}\right)\left(\overline{\kappa_{2}^{ \pm}} \Gamma^{a} \Gamma^{i} \Gamma Q^{-}\right) \\
& -\left(\overline{\kappa_{2}^{ \pm}} \Gamma_{a} E_{i}^{\mp}\right)\left(\overline{\kappa_{1}^{ \pm}} \Gamma^{a} \Gamma^{i} \Gamma Q^{-}\right)
\end{aligned}
$$

where $\theta^{ \pm}=P_{ \pm} \theta$ and the upper sign in a given term in (26) refers to the case $p$ even, while the lower sign refers to the case $p$ odd. The distinction between $p$ even and $p$ odd arises because for $p$ even

$$
P_{ \pm} \Gamma^{i} P_{\mp}=0, \quad P_{ \pm} \Gamma^{\bar{a}} P_{ \pm}=0,
$$

while for $p$ odd,

$$
P_{ \pm} \Gamma^{i} P_{ \pm}=0, \quad P_{ \pm} \Gamma^{\bar{a}} P_{\mp}=0 .
$$

For the case $p=1$, (26) is easily shown to be true if the spacetime gamma matrix identity

$$
\left(C \Gamma^{a}\right)_{\alpha(\beta}\left(C \Gamma_{a}\right)_{\gamma \delta)}
$$


applies (where the round brackets denote the totally symmetric piece). The gamma matrix identity is applied to the second term, and use must be made of the fact that

$$
\left(\overline{\kappa_{2}^{-}} \Gamma_{j} \kappa_{1}^{-}\right)\left(\overline{E_{i}^{+}} \Gamma^{j} \Gamma^{i} \Gamma Q^{-}\right)=\left(\overline{\kappa_{2}^{-}} \Gamma_{j} \kappa_{1}^{-}\right) G^{i j}\left(\overline{E_{i}^{+}} \Gamma Q^{-}\right)+\left(\overline{\kappa_{2}^{-}} \Gamma^{i} \Gamma \kappa_{1}^{-}\right)\left(\overline{E_{i}^{+}} Q^{-}\right)
$$

via the $p=1$ version of the identities

$$
\begin{aligned}
\Gamma^{i} \Gamma & =\frac{i^{\left[\frac{p+1}{2}\right]}}{p !} \epsilon^{i i_{1} \cdots i_{p}} \Gamma_{i_{1}} \cdots \Gamma_{i_{p}} \\
{\left[\Gamma^{i}, \Gamma^{j}\right] \Gamma } & =-2 \frac{i^{\left[\frac{p+1}{2}\right]}}{(p-1) !} \epsilon^{i j i_{2} \cdots i_{p}} \Gamma_{i_{2}} \cdots \Gamma_{i_{p}} .
\end{aligned}
$$

For the case $p>1$, the situation is a little more complicated. The identity (26) can be proved in spacetime dimensions for which the gamma matrix identity

$$
0=\left(C \Gamma_{a_{1}}\right)_{(\alpha \beta}\left(C \Gamma^{a_{1} a_{2} \cdots a_{p}}\right)_{\gamma \delta)}
$$

is true, where $\Gamma^{a_{1} a_{2} \cdots a_{p}}$ is the totally antisymmetric product of $p$ gamma matrices. To apply the gamma matrix identity, we write

$$
\begin{aligned}
\left(\overline{\kappa_{1}^{ \pm}} \Gamma_{\bar{a}} E_{i}^{\mp}\right)\left(\overline{\kappa_{2}^{ \pm}} \Gamma^{\bar{a}} \Gamma^{i} \Gamma Q^{-}\right)= & i^{\left[\frac{p+1}{2}\right]} \frac{1}{p !} \epsilon^{i i_{1} \cdots i_{p}} \\
& \left(\overline{\kappa_{1}^{ \pm}} \Gamma^{a} E_{i}^{\mp}\right)\left(\overline{\kappa_{2}^{ \pm}} \Gamma_{a i_{1} \cdots i_{p-1}} \Gamma_{i_{p}} Q^{-}\right) .
\end{aligned}
$$

If, in addition, the condition

$$
0=\left(P_{-} \Gamma^{i} E_{i}\right)^{\alpha}=\left(\Gamma^{i} E_{i}^{\mp}\right)^{\alpha}
$$

is satisfied, then the gamma matrix identity (after use of (27)) yields

$$
\begin{aligned}
0 & \left.=\left(\overline{\kappa_{1}^{ \pm}} \Gamma_{\bar{a}} E_{i}^{\mp}\right) \overline{\left(\kappa_{2}^{ \pm}\right.} \Gamma^{\bar{a}} \Gamma^{i} \Gamma Q^{-}\right) \\
& -\frac{(p+1)}{p}\left(\overline{\kappa_{2}^{ \pm}} \Gamma^{i} \kappa_{1}^{ \pm}\right)\left(\overline{E_{i}^{\mp}} Q^{-}\right) \\
& -\left(\overline{\kappa_{2}^{ \pm}} \Gamma_{\bar{a}} E_{i}^{\mp}\right)\left(\overline{\left(\kappa_{1}^{ \pm}\right.} \Gamma^{\bar{a}} \Gamma^{i} \Gamma Q^{-}\right) \\
& -\frac{(p+1)}{p}\left(\overline{\kappa_{2}^{ \pm}} \Gamma^{i} \kappa_{1}^{ \pm}\right)\left(\overline{E_{i}^{\mp}} Q^{-}\right) \\
& -\frac{1}{p}\left(\overline{\kappa_{2}^{ \pm}} \Gamma_{\bar{a}} E_{i}^{\mp}\right)\left(\overline{\kappa_{1}^{ \pm}} \Gamma^{\bar{a}} \Gamma^{i} \Gamma Q^{-}\right) \\
& +\frac{1}{p}\left(\overline{\kappa_{1}^{ \pm}} \Gamma_{\bar{a}} E_{i}^{\mp}\right)\left(\overline{\kappa_{2}^{ \pm}} \Gamma^{\bar{a}} \Gamma^{i} \Gamma Q^{-}\right) .
\end{aligned}
$$


This is the required result (26). Note that the identity (26) is not true if the condition is not enforced (28), as otherwise there remain terms containing $\left(\Gamma^{i} E_{i}^{ \pm}\right)^{\alpha}$ which do not cancel against each other.

\section{References}

[1] M. Green and J. Schwarz, Phys. Lett. 136B (1984) 367; Nucl. Phys. B243 (1984) 285.

[2] J.A. Azcárraga and J. Lukierski, Phys. Lett. B113 (1982) 170; Phys. Rev. D28 (1983) 1337.

[3] W. Siegel, Phys. Lett. 128B (1983) 397.

[4] J. Hughes and J. Polchinski, Nucl. Phys. B278 (1986) 147;

[5] J. Hughes, J. Lui and J. Polchinski, Phys. Lett. B180 (1986) 370.

[6] M. Henneaux and L. Mezincescu, Phys. Lett. B152 (1985) 340.

[7] J.A. de Azcárranga and P.K. Townsend, Phys. Rev. 62 (1989) 2579.

[8] R.R. Metsaev and A.A. Tseytlin, Phys. Lett. B436, (1998) 281.

[9] D. Sorokin, V. Tkach and D.V. Volkov, Mod. Phys. Lett. A4 (1989) 901.

[10] S.J. Gates Jr. and H. Nishino, Class. Quantum Grav. 3 (1986) 391; R. Brooks, F. Muhammed and S.J. Gates Jr, Class. Quantum Grav. 3 (1986) 745 .

[11] D. Sorokin, Superbranes and Superembeddings, hep-th/9906142.

[12] M. Green, J. Schwarz and E. Witten, Superstring Theory, Volume 1, Cambridge University Press (1987), Chapter 5.

[13] A. Salam and J. Strathdee, Nucl. Phys. B76 (1974) 477.

[14] J.A. de Azcárraga, J.M. Izquierdo and P.K. Townsend, Phys. Lett. B267 (1991) 366. 
[15] I. Bars, C. Deliduman and D. Minic, Phys. Rev D59 (1999) 125004; Phys. Lett. B457 (1999) 275.

[16] E. Witten, Nucl. Phys. B266 (1986) 245.

[17] M.T. Grisaru, P. Howe, L. Mezincescu, B.E.W. Nilsson and P.K. Townsend, Phys. Lett. 162B (1985) 116.

[18] A. Achucarro, J. Evans, P. Townsend and D. Wiltshire, Phys. Lett. 198B (1987) 441.

[19] A. Salam and J. Strathdee, Ann. Phys. 141 (1982) 316.

[20] R.R. Metsaev and A.A. Tseytlin, Nucl. Phys. B533 (1998) 109.

[21] B. de Wit, K. Peeters and J.C. Plefka, Nucl. Phys. B532 (1998) 99.

[22] A. Salam and J. Strathdee, Nucl. Phys. B76 (1974) 477.

[23] E. Bergshoeff, E. Sezgin and P.K. Townsend, Phys. Lett. B189 (1987) 75; Ann. Phys. 185 (1988) 330.

[24] B. de Wit, K. Peeters, J.C. Plefka and A. Sevrin, Phys .Lett. B443 (1998) 153.

[25] P. Claus, Phys. Rev. D59 (1999) 066003.

[26] See, for example, section 2.2 of the lectures by H. Nicolai in Recent Aspects of Quantum Fields, Proceedings, Schladming 1991, eds. H. Mitter and H. Gausterer, Springer Verlag, 1991.

[27] J. Wess and B. Zumino, Phys. Lett. 37B (1971) 95.

[28] M. Aganagic, C. Popescu and J.H. Schwarz, Phys. Lett. B393 (1997) 311; Nucl. Phys. B495 (1997) 99.

[29] J. Wess and B. Zumino, Phys. Lett. 79B (1978) 394. 